\title{
Evaluation of the Phenotypic and Molecular Pattern of Efflux Pumps in Clinical Isolates of Methicillin-resistant Staphylococcus aureus
}

\author{
Jalal Ghaderkhani ${ }^{1}$, Hamed Tahmasebi ${ }^{2}$, Behruz Zeyni ${ }^{3}$, Sanaz Dehbashi ${ }^{1}$, Mohammad Reza \\ Arabestani ${ }^{4, *}$ \\ ${ }^{1}$ PhD Student, Department of Microbiology, Hamadan University of Medical Sciences, Hamadan, Iran \\ ${ }^{2}$ MSc, Department of Microbiology, Zahedan University of Medical Sciences, Zahedan, Iran \\ ${ }^{3}$ MSc, Department of Microbiology, Hamadan University of Medical Sciences, Hamadan, Iran \\ ${ }^{4}$ Associate Professor, Nutrition Health Research Center, Hamadan University of Medical Sciences, Hamadan, Iran
}

* Corresponding Author: Mohammad Reza Arabestani, Department of Microbiology, Hamadan University of Medical Sciences, Hamadan, Iran. Email: mohammad.arabestani@gmail.com

\section{Abstract}

Received: 16.05 .2017 Accepted: 10.09.2017

How to Cite this Article: Ghaderkhani J, Tahmasebi H, Zeyni B, Dehbashi S, Arabestani MR. Evaluation of the Phenotypic and Molecular Pattern of Efflux Pumps in Clinical Isolates of Methicillin-resistant Staphylo-coccus aureus. Sci J Hamadan Univ Med Sci. 2017; 24(3): 183-191. DOI: $10.18869 /$ acadpub.ajcm.24.3.183.
Background and Objective: Efflux pumps are regarded as one of the most important mechanisms of antibiotic resistance in this era. Staphylococcus aureus is one of the bacterial groups with efflux pumps. The pumps' activity is coded by specific genes. The aim of this study was to determine the phenotypic and molecular pattern of efflux pumps in the clinical isolates of methicillin-resistant $S$. aureus.

Materials and Methods: This study was conducted on 302 S. aureus bacteria collected from different clinical specimens. We detected 145 isolates of methicillin-resistant using disk diffusion method with cefoxitin $(30 \mu \mathrm{g})$ as well as minimum inhibitory concentration with E-test strips and cefoxitin disks. In addition, multiplex polymerase chain reaction method was employed to identify the genes responsible for resistance to efflux pump and gyrase. The data were analyzed using SPSS software version 16.

Results: Among the 145 isolates of methicillin-resistant $S$. aureus, norfloxacin and ciprofloxacin had the highest frequency. Furthermore, norA, norB, nor $C$, grlA, $g r l B, g y r A$, and $g y r B$ genes were positive in 39 (25.825\%), $12(9.97 \%)$, $41(49.15 \%), 75(49.66 \%), 37(26.50 \%), 58(38.41 \%)$, and 19 clinical isolates $(12.58 \%)$, respectively.

Conclusion: As the findings indicated, the presence of efflux pumps in the strains of methicillin-resistant Staphylococcus aureus provided the ground for the emergence of multi-drug resistant bacteria.

Keywords: Efflux Pumps; Fluoroquinolone; Resistant to Methicillin; Staphylococcus aureus 
dof: 10.18869 /acadpub.ajcm.24.3.183

\title{
ارزيابى شيوع فنوتيّى و مولكولى يڤֶ هاى افلاكس در ايزوله هاى بالينى استافيلوكوى اورئوس مقاوم به متى سيلين
}

\author{
جلال قادر خانى'، حامد طهماسبى '، بهروز زينى '، ساناز ده باشى'، محمد رضا عربستانى ؟.* \\ ' دانشجوى دكتراى تخصصى، گروه ميكروب شناسى، دانشكده يزشكى، دانشكاه علوم يزشكى همدان، همدان، ايران

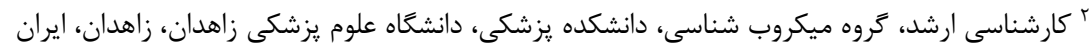

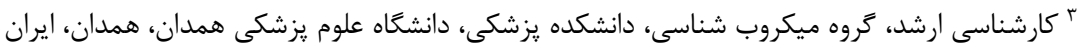

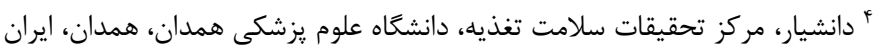

* نويسنده مسئول: محمد رضا عربستانى، گروه ميكروبشناسى، دانشكده يزشكى، دانشكاه علوم يزشكى همدان، همدان، ايران. ايميل: mohammad.arabestani@gmail.com

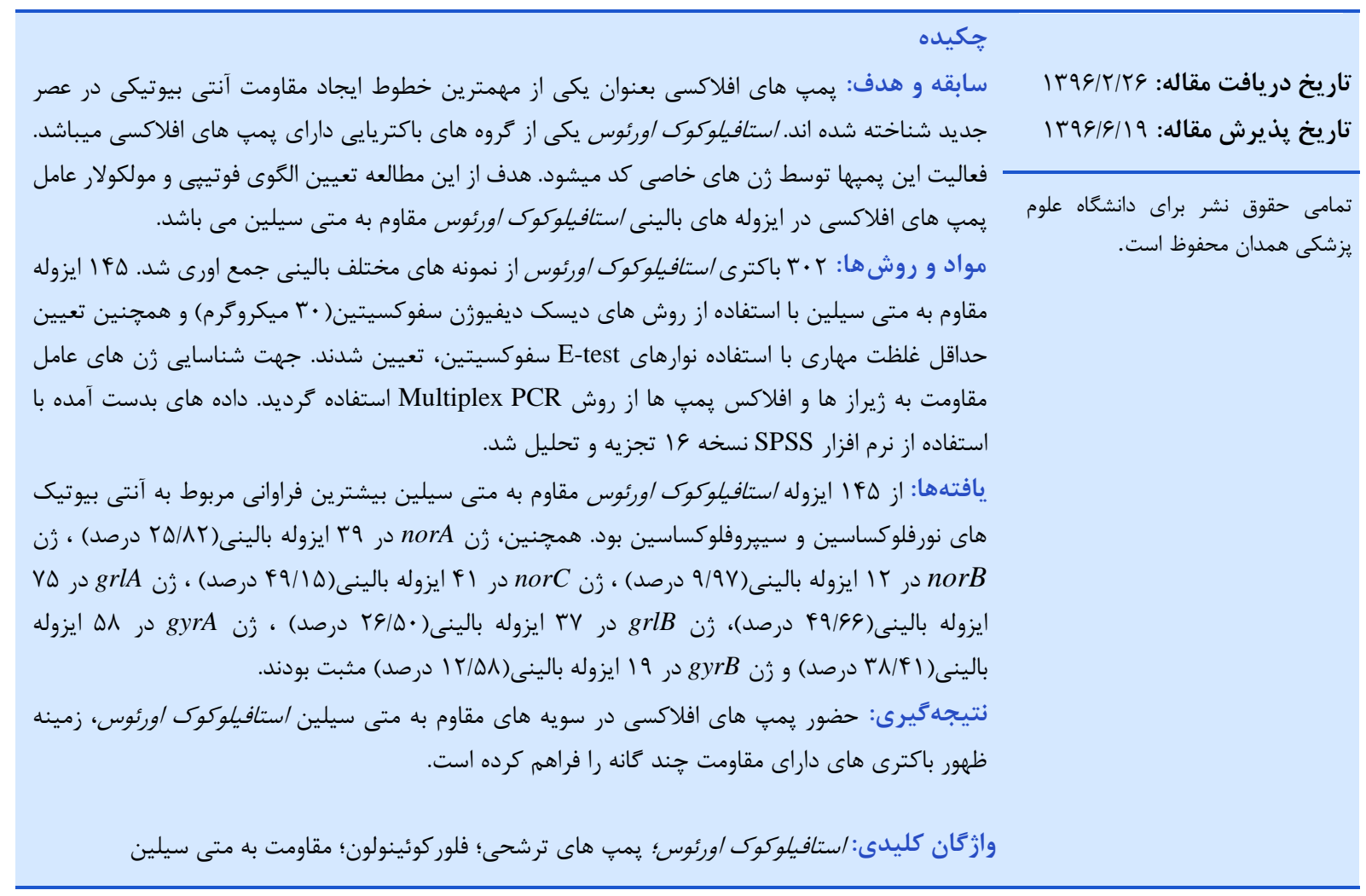

مقدمه

نايايدار، ز ن هاى وابسته به كد شدن اين پِمٍ ها را فعال كرده،

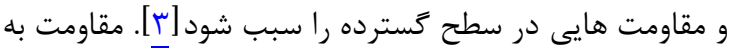

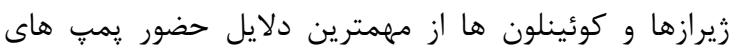

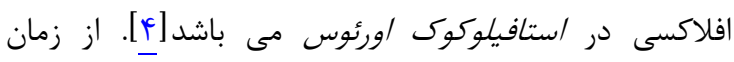

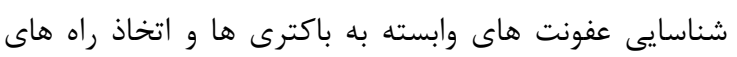

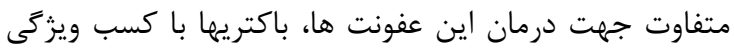

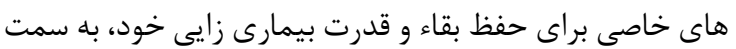
تكامل و مقاوم شدن در برابر طيف خسترده ايى از آنتى بيوتيك حفي

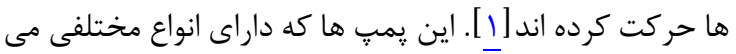

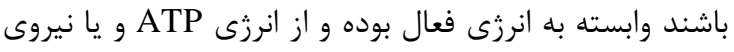

يمب هاى افلاكسى به عنوان يكى از مههم ترين خطوط

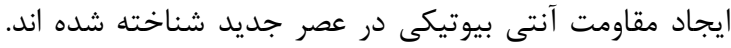

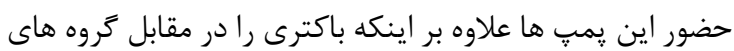
مختلف آنتى بيوتيكى مقاوم مى كنيند، ميى توانند سبب بـ بروز

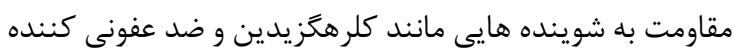

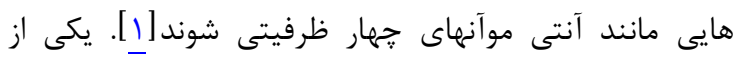

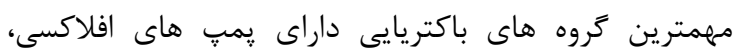

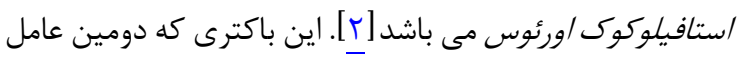

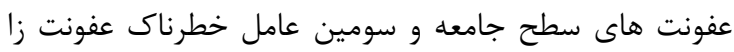

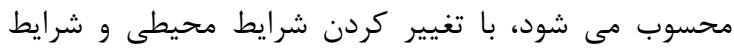


صورت كرفت، r •r نمونه بالينى شامل خون، ادرار، كاتاتر، سواب بينى، زخم، ترشحات، تراشه، خلط، بزاق و ساير موارد، از بيماران

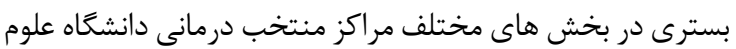

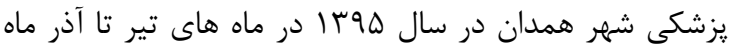

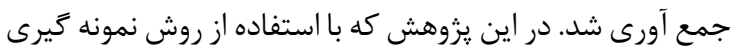

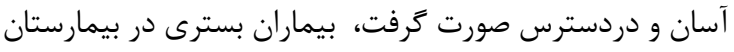

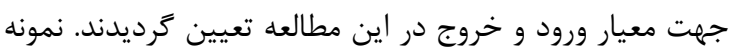

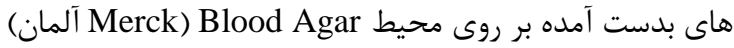

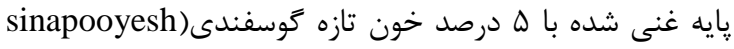

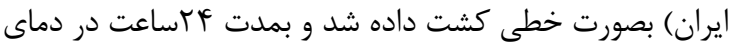
Vاسر جه انكوبه شد. تست هاى تشخيصى با توجه به يروتكل هاى دئي

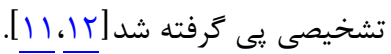

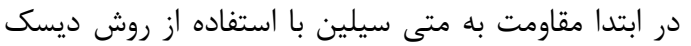

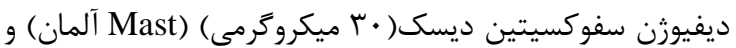

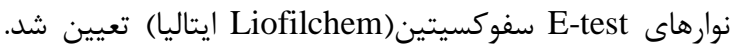
الكوى مقاومت آنتى بيوتيك به انتى بيوتيك هاى برى براى تعيين

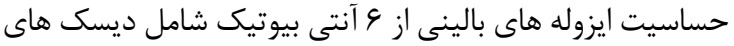

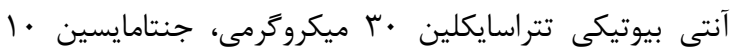

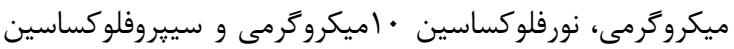
هميكروگرمى، كاتى فلوكساسين ه ميكروكرمى و و اوفلوكساسين

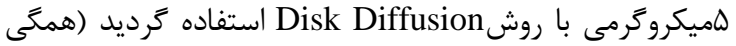

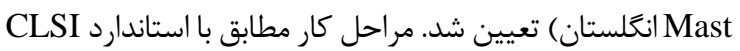

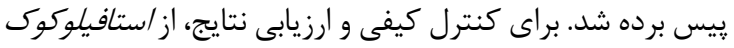

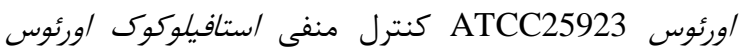

ATCC43300 بعنوان منترل مثبت استفاده شد [ ا I] براى انجام استخراج DNA رنوميك از كيت استخراج شركت منتبن

سينا زن ايران بر اساس :رتكل شركت سازنده انجام شد. DNA

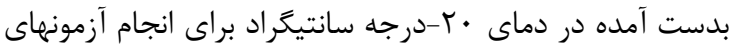
مولكولى، ذخيره شد [ب I]].

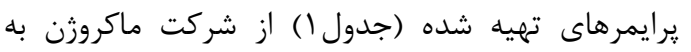
سفارش پِيشگام ايران براى شناسايى زنهاى gyrA، gyrB، norC،norB ،norA ،grlB،grlA كرفت. حجم واكنش در ه r ميكروليتر كه شامل זميكروليتر از
انتقال يروتون استفاده مى كنند. افلاكس پِمٍ هاى آنتى بيوتيكى

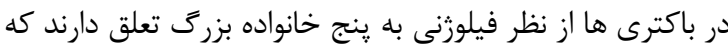

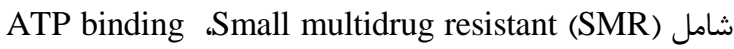
،Resistant nodulation divition (RND) ،(ABC) cassette Multidrug and Major facilitator superfamily (MFS) (MATE) toxic efflux ABC

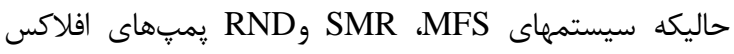

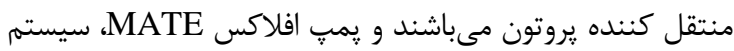

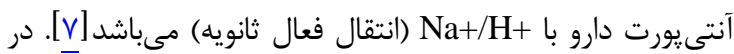
كنار مقاومت هاى آنتى بيوتيكى حاصل از افلاكس يمبٍ ها ، إين

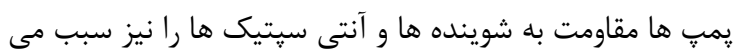

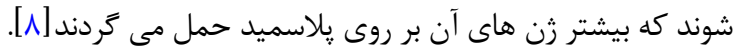

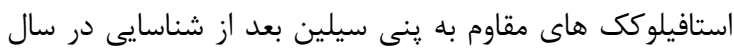

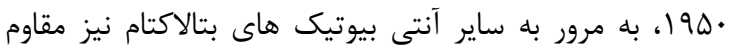

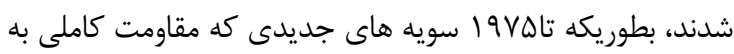

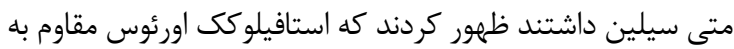

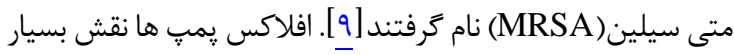
مهمى در ظهور سويه هاى استافيلوكى اورئوس داراى مقاومت

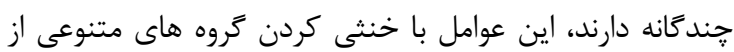

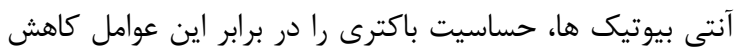

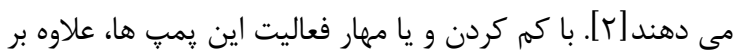

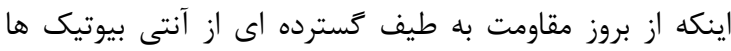
جلوگيرى مى شود، مى توان مانع بروز مقاومت به شوينده ها و

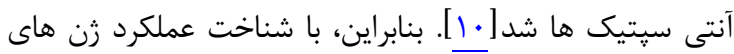

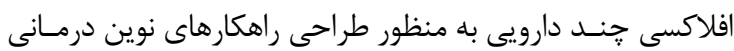

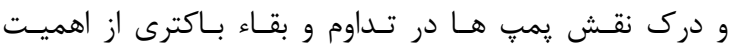

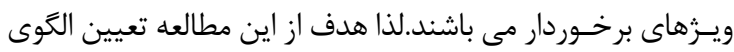

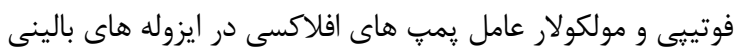
استافيلوكوك /ورئوس مقاوم به متى سيلين مى بلى باشد.

مواد و روشها

در اين مطالعه توصيفى- تحليلى، كه طى يك دوره 9 ماهه

جدول ا: توالى هاى نوكلئوتيدى مورد استفاده جهت شناسايى عوامل مقاومت به فلوروكوئينولونى

\begin{tabular}{|c|c|c|c|}
\hline ثن مالى مورد & يرايمر هاى مورد & توالى هاى نوكلئوتيدى & $\begin{array}{r}\text { (جول آميليكون باز) } \\
\text { (جفون }\end{array}$ \\
\hline $\operatorname{grlB}$ & $\begin{array}{l}\text { grlB-1 } \\
\text { grlB-2 }\end{array}$ & $\begin{array}{l}\text { CGATTAAAGCACAACAAGCAAG } \\
\text { CATCAGTCATAATAATTACTC }\end{array}$ & rvd \\
\hline gyrA & $\begin{array}{l}\text { gyrA-1 } \\
\text { gyrA-2 }\end{array}$ & $\begin{array}{l}\text { AATGAACAAGGTATGACACC } \\
\text { TACGCGCTTCAGTATAACGC }\end{array}$ & TMT \\
\hline gyr $B$ & $\begin{array}{l}\text { gyrB-1 } \\
\text { gyrB-2 }\end{array}$ & $\begin{array}{l}\text { CAGCGTTAGATGTAGCAAGC } \\
\text { CCGATTCCTGTACCAAATGC }\end{array}$ & $r \Delta$. \\
\hline $\operatorname{grl} A$ & $\begin{array}{l}\text { grl-a } \\
\text { grl-b }\end{array}$ & $\begin{array}{c}\text { GATGTTATGGTCAATATCATCCA } \\
\text { AAGAAACTGTCTCTTTATTAATATCACGT }\end{array}$ & T19 \\
\hline norA & $\begin{array}{l}\text { norA-F } \\
\text { norA-R }\end{array}$ & $\begin{array}{l}\text { TTTGTTTTCAGTGTCAGAATTTATGTTTG } \\
\text { GGCTTGGTGAAATATCAGCTATTAAAC }\end{array}$ & If. \\
\hline nor $B$ & $\begin{array}{l}\text { norB-F } \\
\text { norB-R }\end{array}$ & $\begin{array}{l}\text { GTAATGGTACTAATTATGATTCGTGTTGG } \\
\text { CTGGCAAGAAAGTTAATGAAATGAGAC }\end{array}$ & . \\
\hline
\end{tabular}


دو براى مقايسه يافته هاى كيفى و تست مثبت مستقل براى

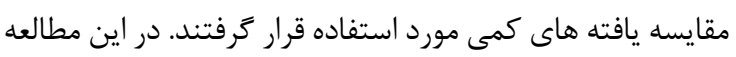

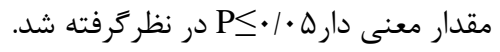

بافته ها

در مجموع |ه| ايزوله /ستافيلوكوكى /ورئوس مقاوم به متى له

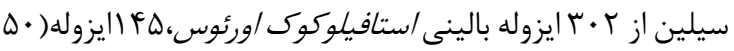

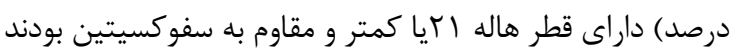
كه با توجه به CLSI به عنوان سويه هاى مقار مقاوم به متى سيلين

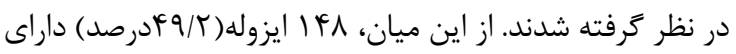

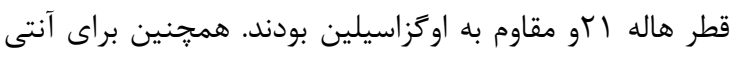

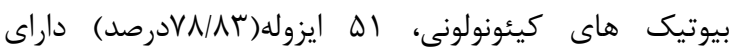

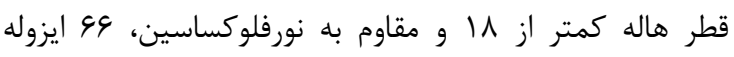

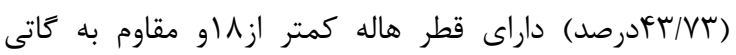

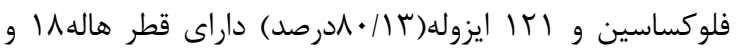
مقاوم به اوفلوكساسين بودند (شكل r، ().

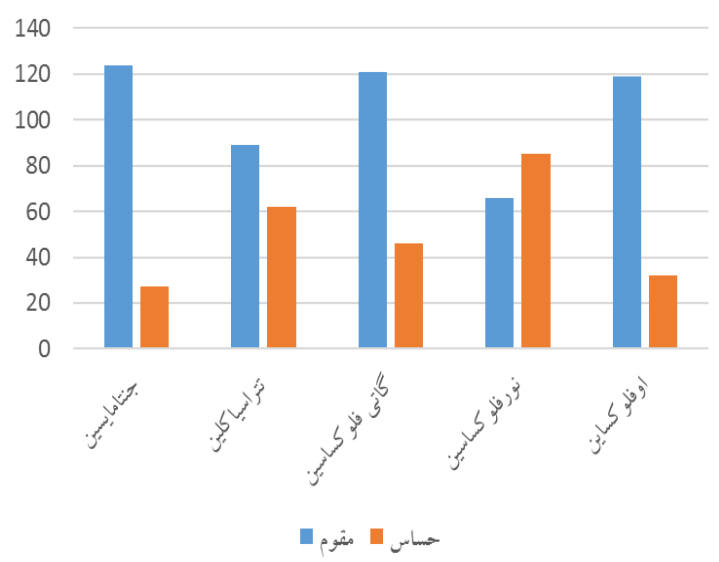

شكل ا: فراوانى الكوى مقاومت آنتى بيوتيكى ايزوله هاى بالينى

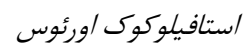

DNA الكَ، اميكروليتر از هر يرايمر با غلظتهآييكومولار و ه, זاميكروليتر از Ampliqon) MasterMixe Tris-Hcl PH8.5, ( $\left.\mathrm{NH}_{4}\right) \mathrm{SO}_{4}, 3 \mathrm{mMMgcl}_{2}$, 0/2 unit Ampliqon ،0/4MmdNTP 0/2\% Tween20 (Insert red dye and stabilizer ،polymeras حجم باقيمانده با اب مقطر ديونيزه به حجم مورد نظر رسانده

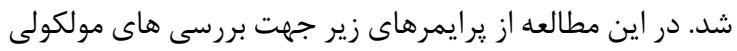

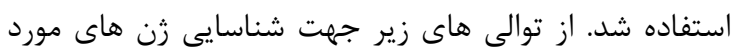

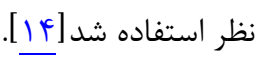

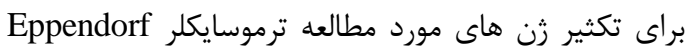

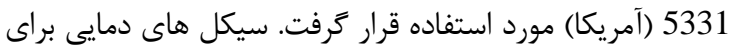

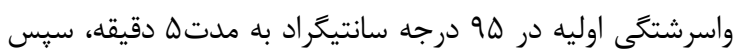

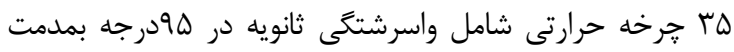

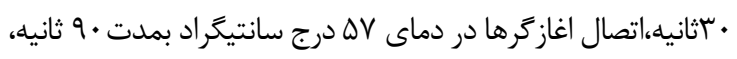

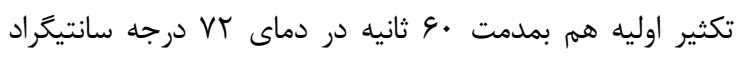

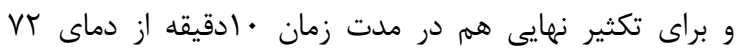

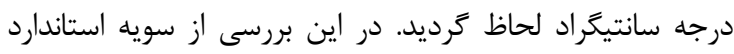

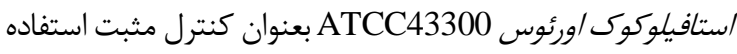

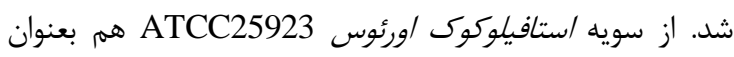
كنترل منفى استفاده شد.

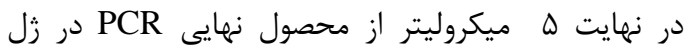

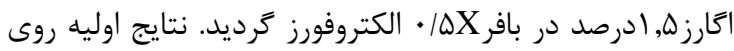

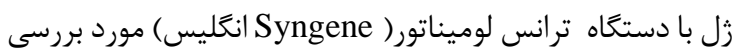

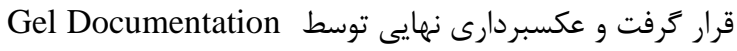

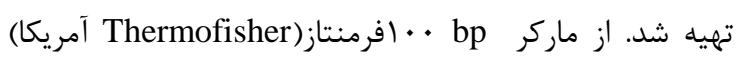
براى شناسايى باند مورد نظر استفاده شد.

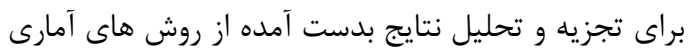

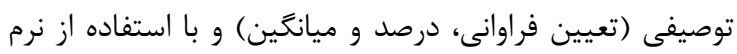

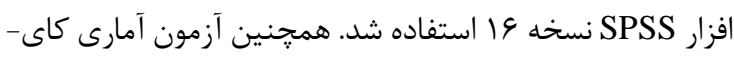

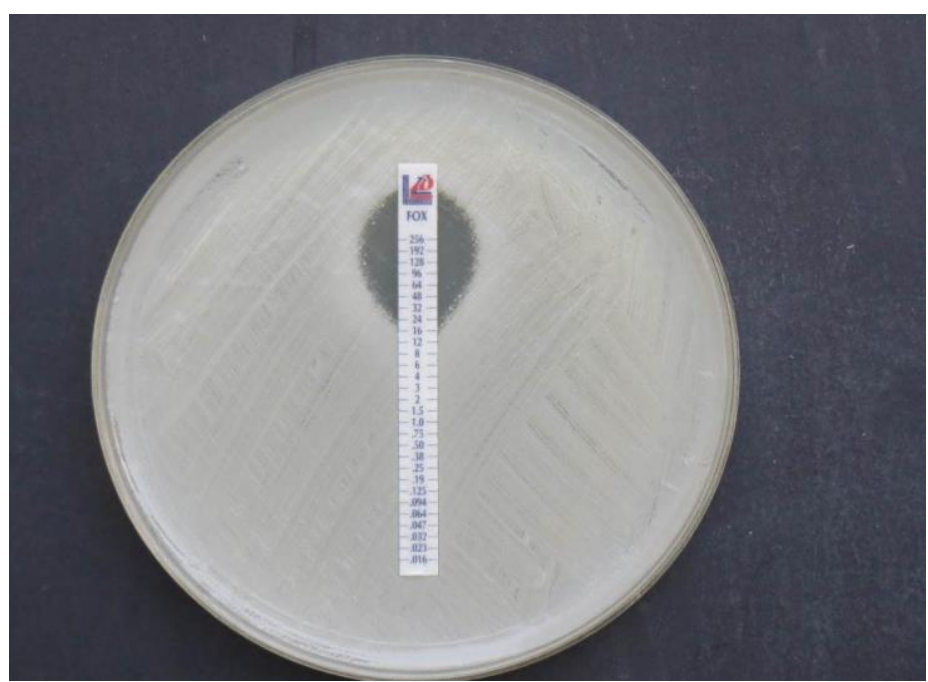

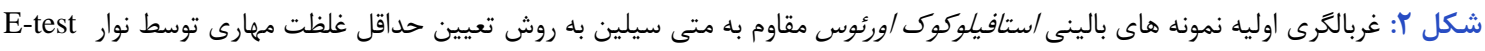
نefoxitin

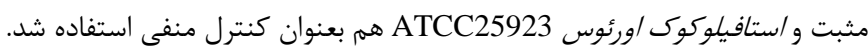


درصد) ، زن grlA در ال ايزوله بالينى(

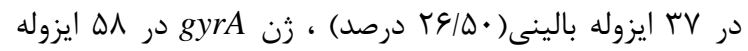

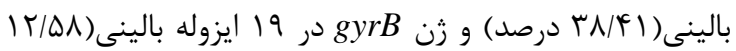
درصد) مثبت بودند (شكل r - (D).
فراوانى زن هاى وابسته به عوامل مقاومتى به فلوروكوئينولون

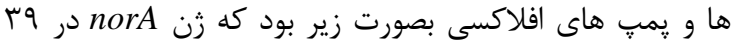
ايزوله بالينى (

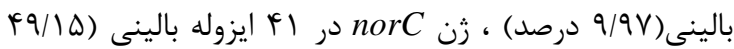

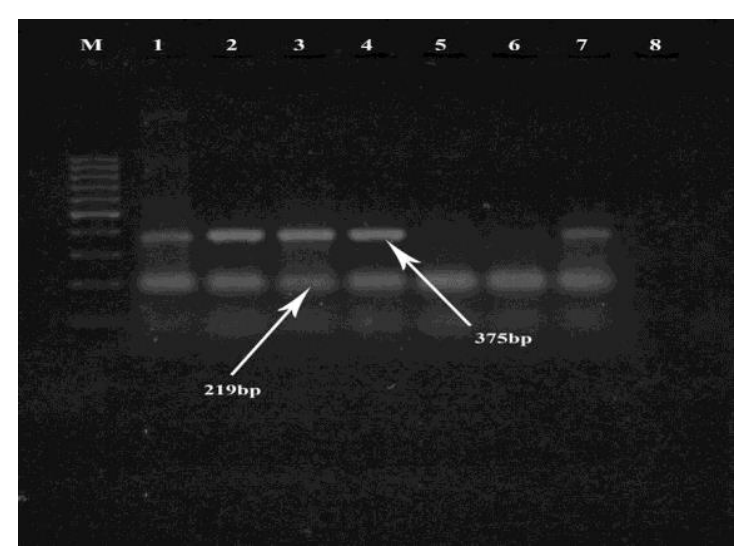

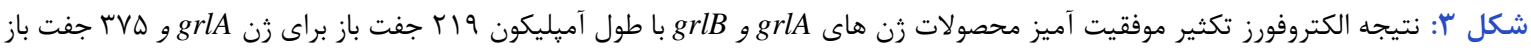

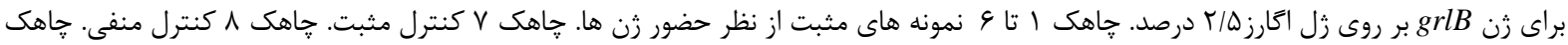
M

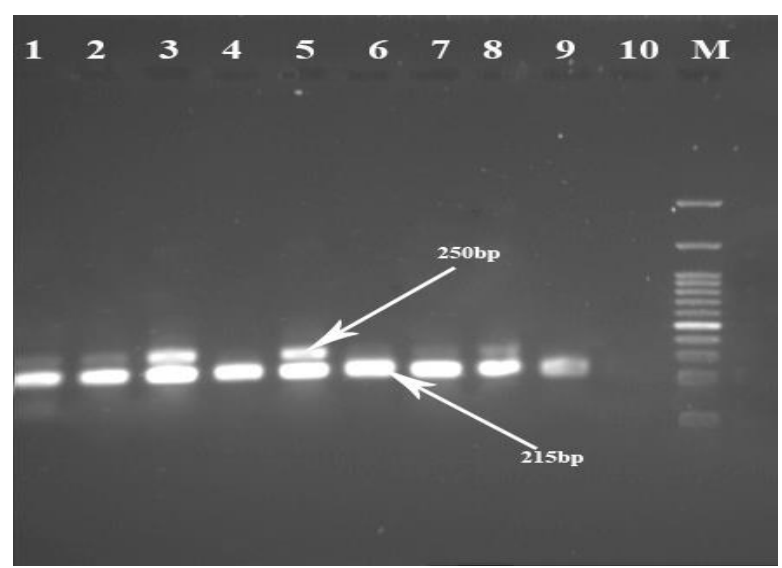

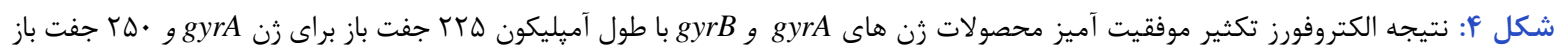

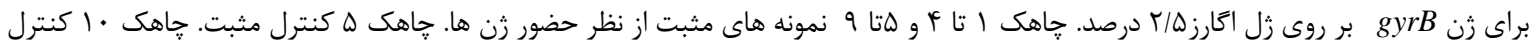

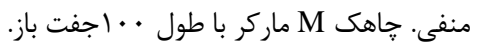

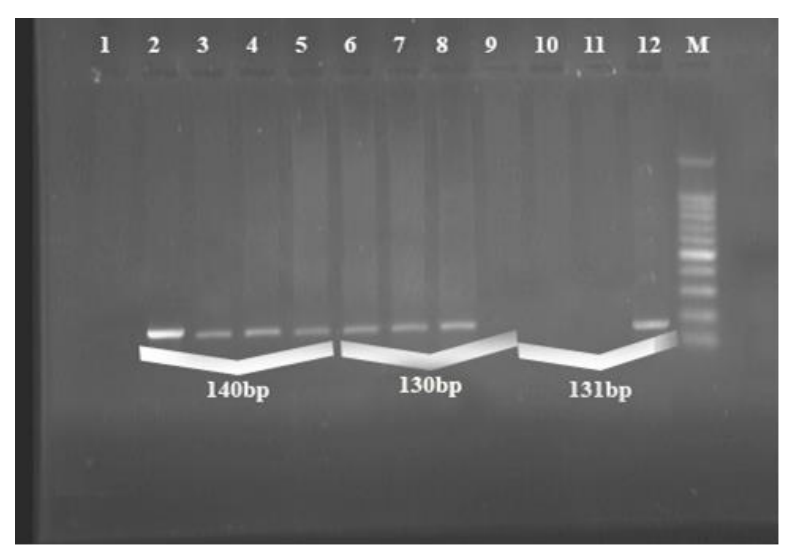

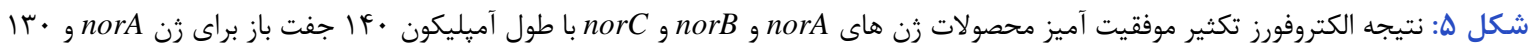

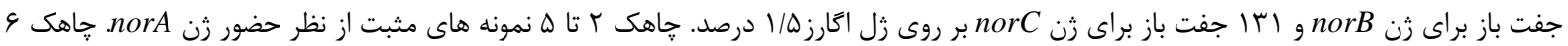

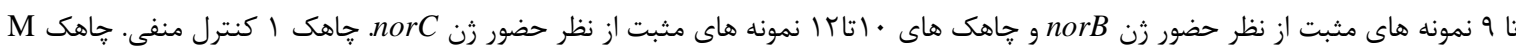

$$
\text { ماركر با طول · • إجفت باز. }
$$


بيوتيكى و رن هاى عامل افلاكسى مشاهده شد. براى همه

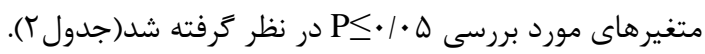

با توجه به فراوانى سويه هاى بدست آمده و نتايج حاصل از تحليل هاى آمارى، ارتباط معنى دارى بين الخوى مقاومت آنتى

جدول זّ: آناليز آمارى بررسى احتمال ارتباط بين الكَى مقاومت آنتى بيوتيكى و يمٍ هاى افلاكس در جدايه هاى بالينى استافيلوكوى اورئوس مقاوم

\begin{tabular}{|c|c|c|c|c|c|c|c|c|c|c|}
\hline قبوتيكى آنتى & $\begin{array}{c}m e c A \\
(\mathbf{n}=\mid f \Delta)\end{array}$ & $\begin{array}{c}(n=1) \\
n o r C \\
(n=F \mid)\end{array}$ & $\begin{array}{c}\text { norB } \\
(n=1 /)\end{array}$ & $\begin{array}{c}\text { norA } \\
\text { مقاوم }(n=r q)\end{array}$ & $\begin{array}{l}\text { كوى اورئز } \\
\text { gyrB } \\
(n=19)\end{array}$ & $\begin{array}{c}\text { اس استافي } \\
\text { gyrA } \\
(\mathbf{n}=\Delta \Lambda)\end{array}$ & $\begin{array}{c}\text { جدايه } \\
\text { grlB } \\
(n=r V)\end{array}$ & $\begin{array}{c}g r l A \\
(\mathbf{n}=\vee \Delta)\end{array}$ & غلظت آنتى & بيوتيكها \\
\hline $\mathrm{R} \leq \ \wedge \mathrm{mm}$ & $P \leq . / \cdot 11$ & $P \leq I \cdot F \varphi$ & $P \leq .1 \cdot 19$ & $P \leq 1 \cdots q$ & $P \leq / \cdot 1 \mu$ & $P \leq .1 \cdot \cdot 1$ & $P \leq .1 \cdot \vee q$ & $P \leq . / \cdot r \varphi$ & ه (ميكرو & نورفلو كساسين \\
\hline $\mathrm{R} \leq \wedge \wedge \mathrm{mm}$ & $P \leq . / \cdot 1$. & $P \leq . l \cdot F V$ & $P \leq . / \cdot \curlyvee \wedge$ & $P \leq 1 \cdot \vee q q$ & $P \leq . / \cdot \varphi^{\prime}$ & $P \leq . / \cdot \omega$ & $P \leq .1 \cdot 19$ & $P \leq .1 \cdot \cdot q$ & ه (ميكرو & كاتى فلوكساسين \\
\hline $\mathrm{R} \leq l \uparrow \mathrm{mm}$ & $P \leq . \mid+\psi_{1}$ & $P \leq / \cdot \bullet \Delta$ & $P \leq . / \cdot \Delta$ & $P \leq / \cdot r r$ & $P \leq . \cdot \cdots \varphi$ & $P \leq . / \cdots \wedge$ & $P \leq . / \cdot \Delta$ & $P \leq . / \cdot Y F$ & • ץ (ميكرو گرم) & تتراسايكلين \\
\hline $\mathrm{R} \leq \ \wedge \mathrm{mm}$ & $P \leq . / \cdot Y \wedge$ & $P \leq .1 \cdot \vee q$ & $P \leq . . \cdot \varphi$ & $P \leq . \mid \cdot 11$ & $P \leq .1 \cdot 19$ & $P \leq . \mid \cdot \cdot 1$ & $P \leq .1 \cdot \cdot 1$ & $P \leq . / \cdot 1 T$ & ه (ميكرو & اوفلو كساسين \\
\hline $\mathrm{R} \leq ı \mathrm{~mm}$ & $P \leq / \cdot r G$ & $P \leq . / \cdot \vee V$ & $P \leq . / \cdots \wedge$ & $P \leq . \mid \cdot \cdot 1$ & $P \leq .1 \cdot 19$ & $P \leq .1 \cdot 19$ & $P \leq . / \cdot \wedge$ & $P \leq . / \cdot r r$ & 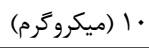 & جنتامايسين \\
\hline
\end{tabular}

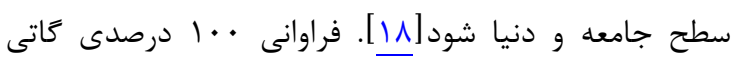

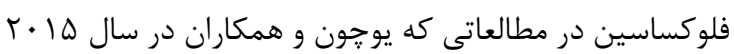

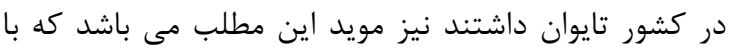

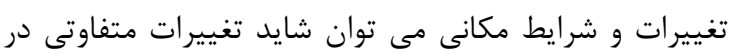

نتايج بدست آمده بود [19]. از مجموع الها سويه مقاوم به متى سيلين فراوانى زن رداد

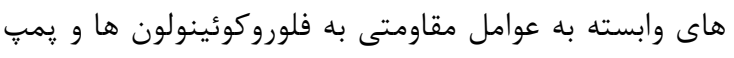

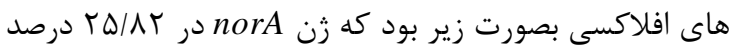

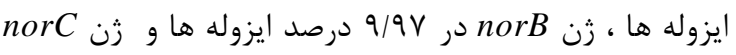

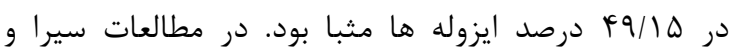

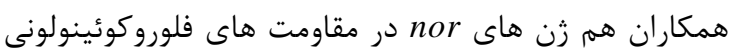

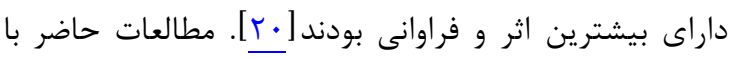

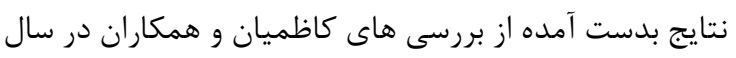

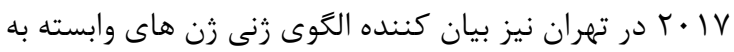

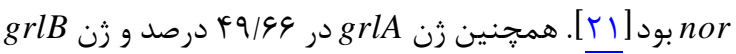

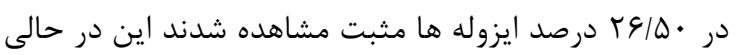

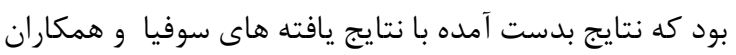

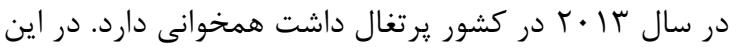

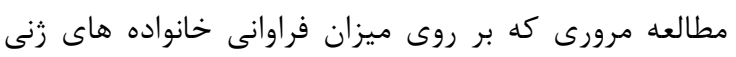

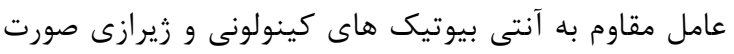

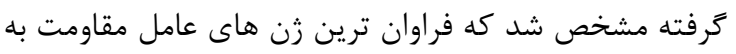

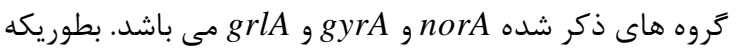

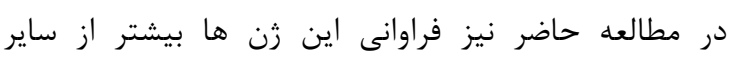

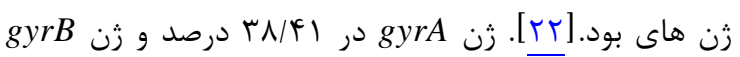

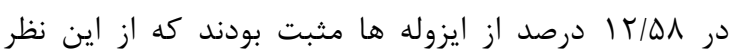

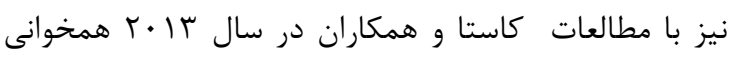

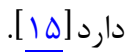
سويه هاى مختلف استافيلوكوك اورئوس كه از نمونه هاى بالينى بدست آمده اند، در بسيارى از موارد خانواده هاى يمب

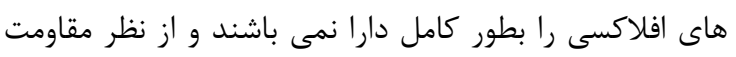

استفاده ازفلوروكينولون ها به عنوان ريسك فاكتورى براى

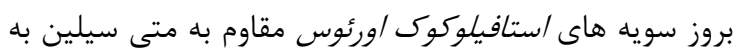

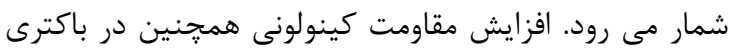

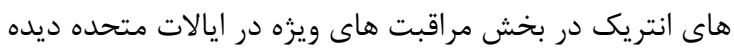

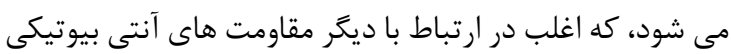

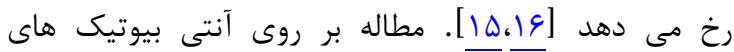
فلوروكوئينولونى در كنار ساير آنتى بيوتيك هاي و مون موثر بر روند

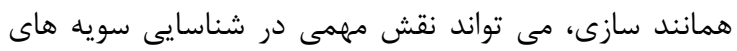

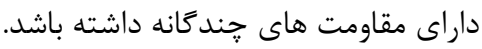

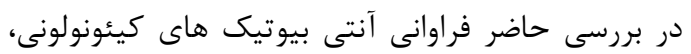

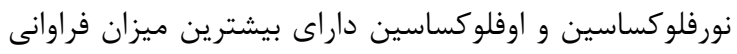
بودند. در مطالعاتى كه احمدى و و همكاران در تهران انجام

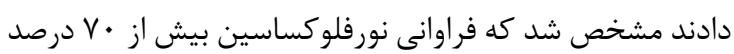

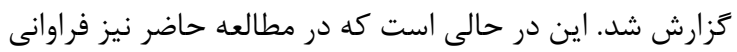

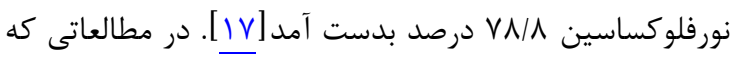

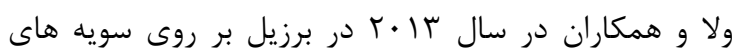

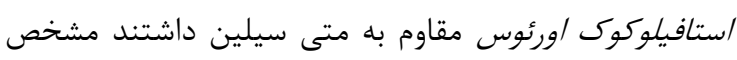

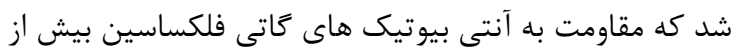

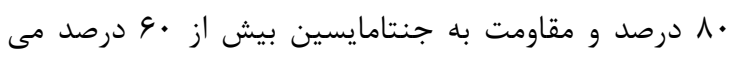

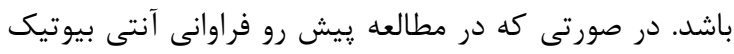

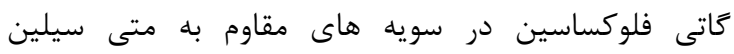

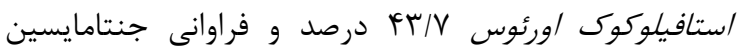

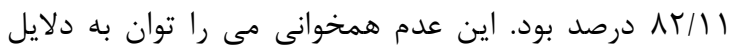

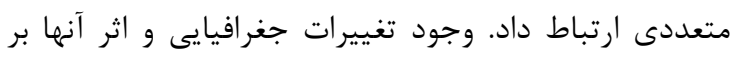
سويه هاى بدست آمده، نوع نمونه اى كه باكترى از آن آن ايزوله

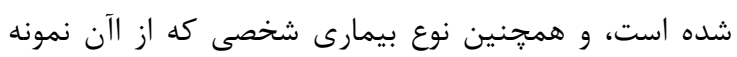

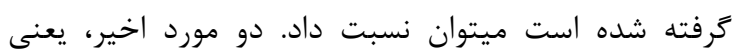

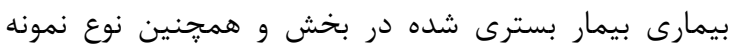

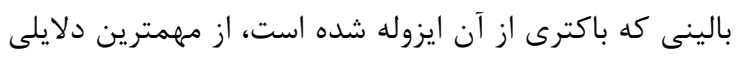

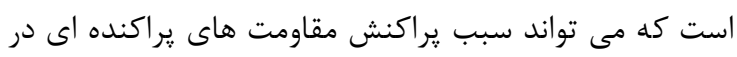




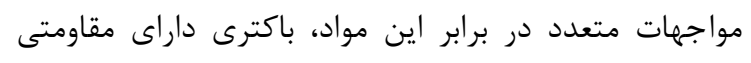

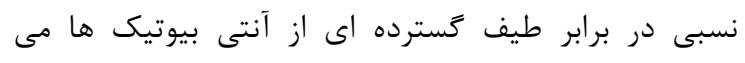

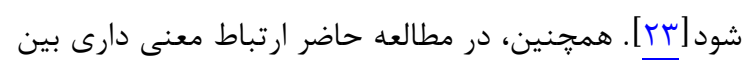

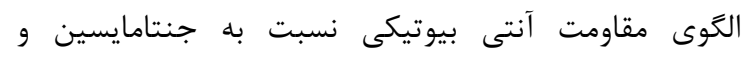
تتراسايكلين نيز مشاهده شد. بطوريكه حضور زن هن هاى gor

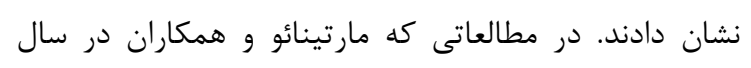

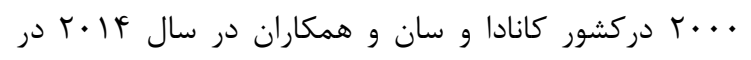

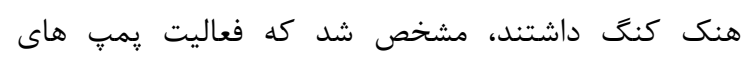

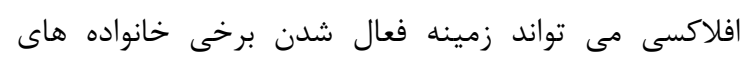

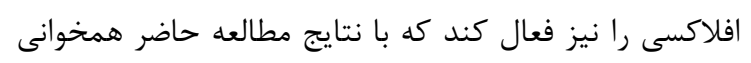

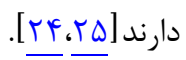

اين نكته را نبايد از ذهن دور كرده كه كَاهى مى تونى توان بين

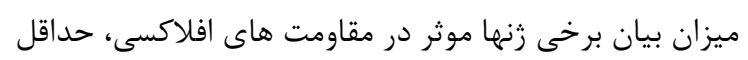

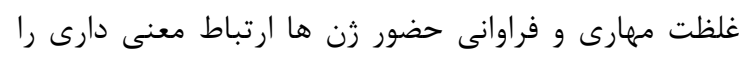

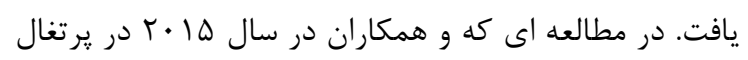

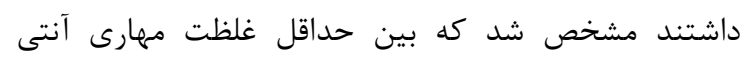

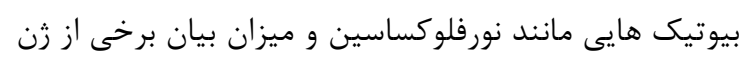

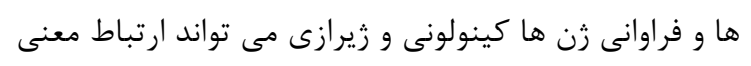

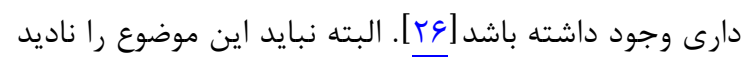

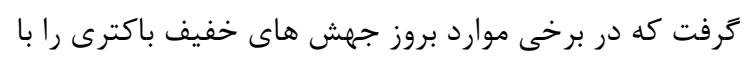

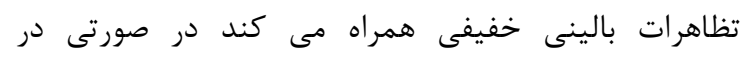

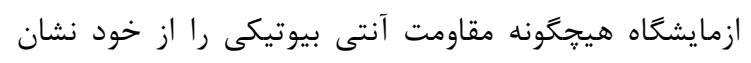

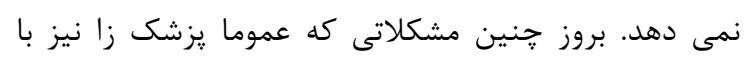

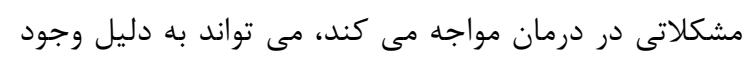

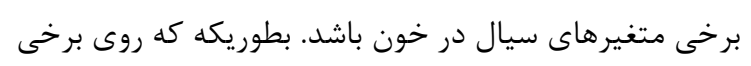

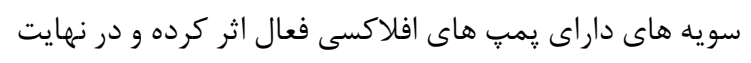
سبب مقاومت نسبى در بالين و حساسيت سويه دائ در آلآمايشعاه

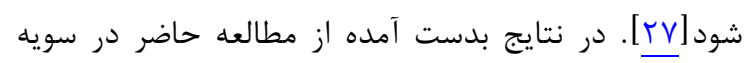

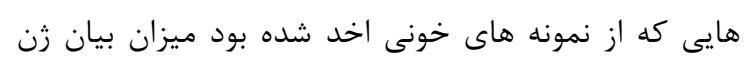

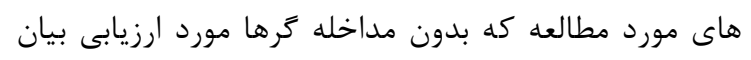

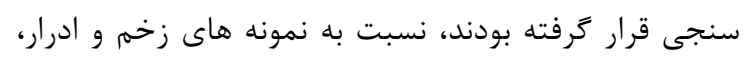

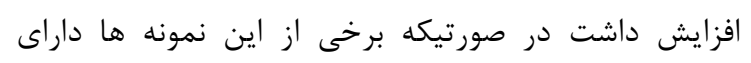

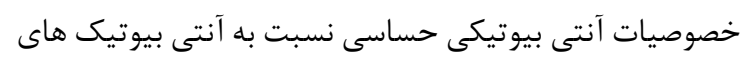

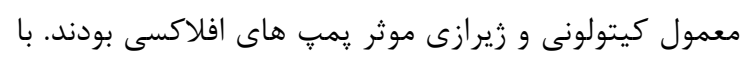

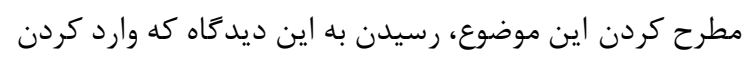

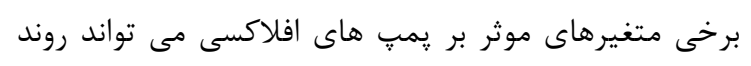

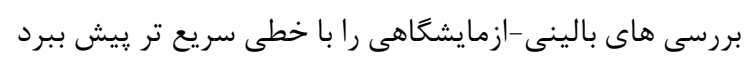
را به ميان مى آورد.

\section{نتيجهَيرى}

سويه هاى/ستافيلوكوك /ورئوس مقاوم به متى سيلين كه به

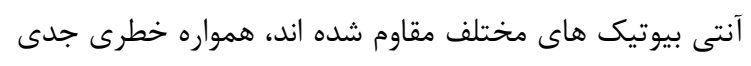

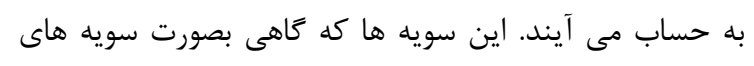

هاى سطح بالا به جند آنتى بيوتيك فلوروكوئينولونى، فراوانى

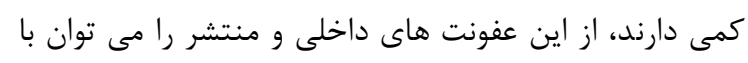

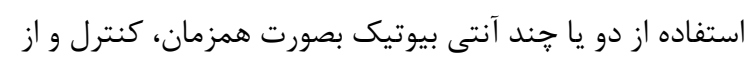

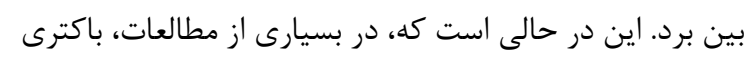

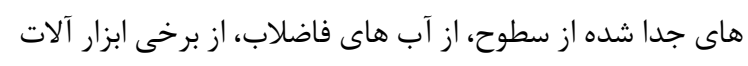

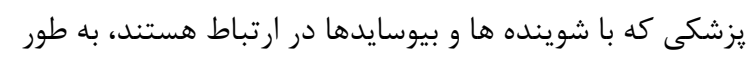

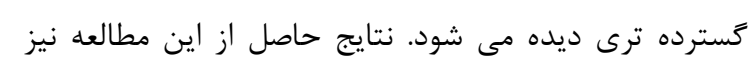

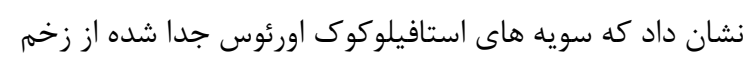

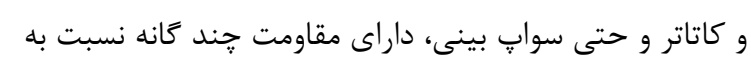

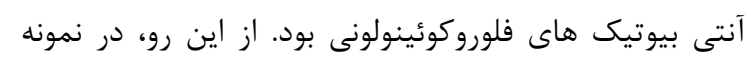

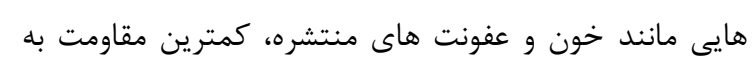

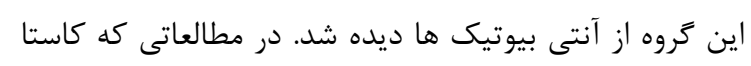

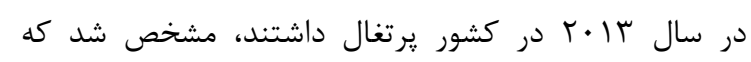

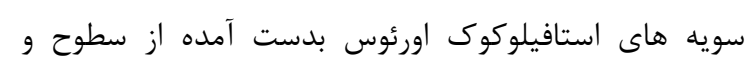

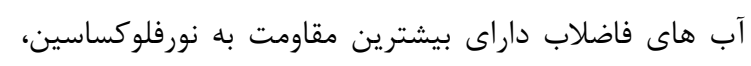

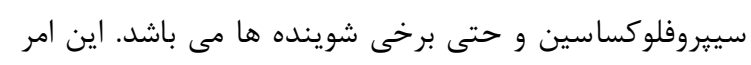

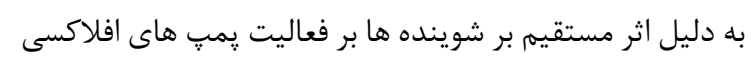

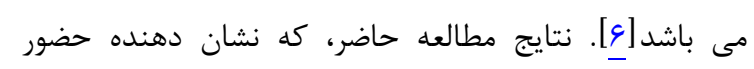

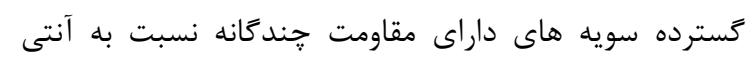

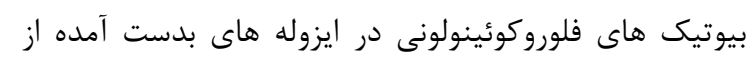

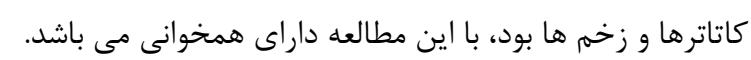

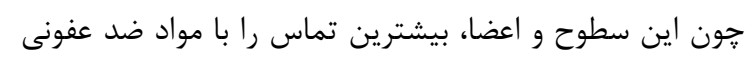

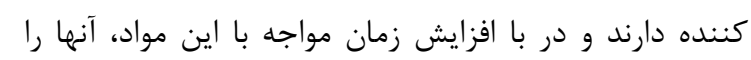

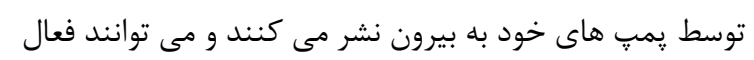

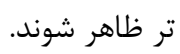
اطلاعات حاصل از اين مطالعه نشان داد كه، بين الكَوى

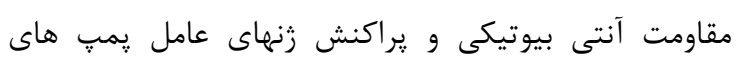

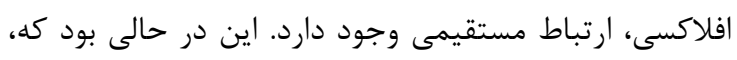

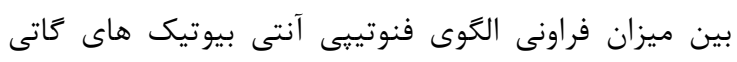

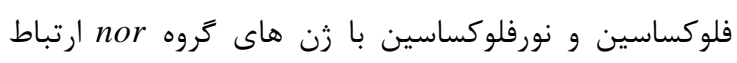

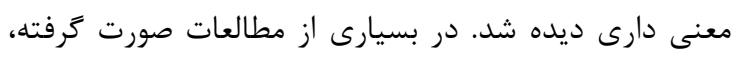

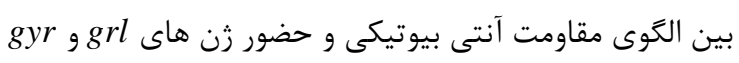

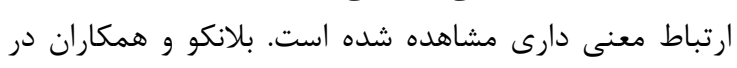

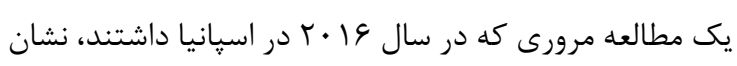

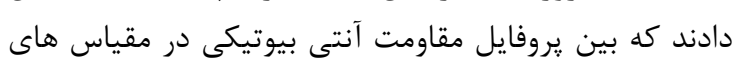

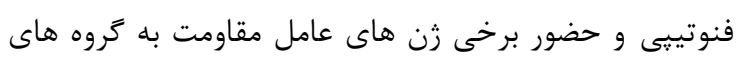

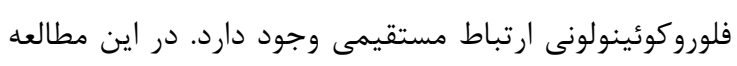

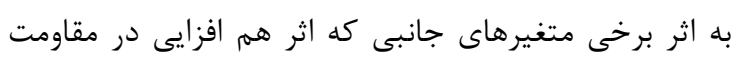

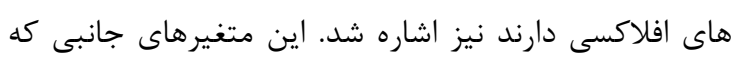

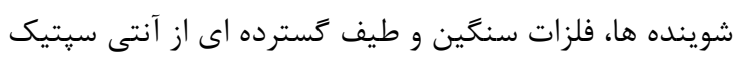

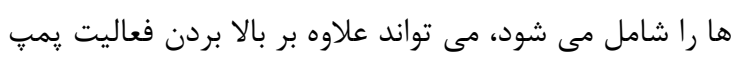

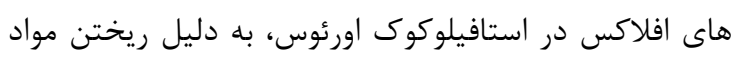

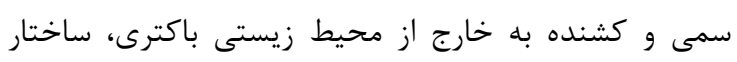

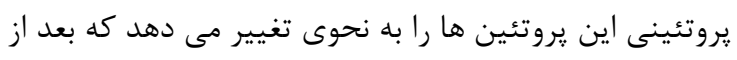




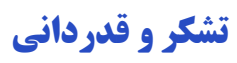

اين مقاله حاصل طرح دانشجويى در سال ه9 1 با شماره

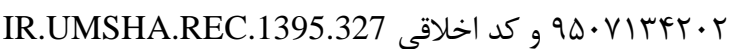
كه با حمايت مالى معاونت يزوهشى دانشعاه علوم يزشكى همدان

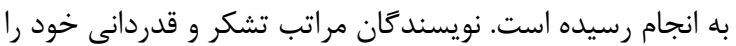

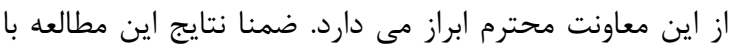

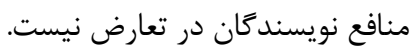

\section{REFERENCES}

1. Tintino SR, Oliveira-Tintino CDM, Campina FF, Silva RLP, Costa MDS, Menezes IR, et al. Evaluation of the tannic acid inhibitory effect against the NorA efflux pump of Staphylococcus aureus. Microb Pathog. 2016;97:9-13. PMID: 27057677 DOI: 10.1016/j.micpath.2016.04.003

2. Couderc C, Thiébaut AC, Lawrence C, Bouchiat C, Herrmann $\mathrm{JL}$, Salomon J, et al. Fluoroquinolone impact on nasal methicillin-resistant and methicillin-sensitive staphylococcus aureus colonization durations in neurologic long-term-care facilities. Antimicrob Agents Chemother. 2015;59(12):7621-8. PMID: 26416866 DOI: 10.1128/AAC.01338-15

3. Zarei Koosha R, Mahmoodzadeh Hosseini H, Mehdizadeh Aghdam E, Ghorbani Tajandareh S, Imani Fooladi AA. Distribution of tsst-1 and mecA genes in staphylococcus aureus isolated from clinical specimens. Jundishapur $J$ Microbiol. 2016;9(3):290-57. PMID: 27226873 DOI: 10.5812/jjm.29057

4. Greninger AL, Chatterjee SS, Chan LC, Hamilton SM, Chambers HF, et al. Whole-genome sequencing of methicillin-resistant staphylococcus aureus resistant to fifthgeneration cephalosporins reveals potential non-meca mechanisms of resistance. PLoS One. 2016;11(2):e0149541. PMID: 26890675 DOI: 10.1371/journal.pone.0149541

5. Kaatz GW, DeMarco CE, Seo SM. MepR, a repressor of the Staphylococcus aureus MATE family multidrug efflux pump MepA, is a substrate-responsive regulatory protein. Antimicrob Agents Chemother. 2006;50(4):1276-81. PMID: 16569840 DOI: 10.1128/AAC.50.4.1276-1281.2006

6. Costa SS, Junqueira E, Palma C, Viveiros M, Melo-Cristino J, Amaral $\mathrm{L}$, et al. Resistance to antimicrobials mediated by efflux pumps in staphylococcus aureus. Antibiotics. 2013;2(11):8399. PMID: 27029294 DOI: $10.3390 /$ antibiotics2010083

7. Khan A, Sultan A, Tyagi A, Zahoor S, Akram M, Kaur S, et al. Amplification of mecA gene in multi-drug resistant Staphylococcus aureus strains from hospital personnel. $J$ Infect Dev Ctries. 2007;1(3):289-95. PMID: 19734607

8. Truong-Bolduc QC, Bolduc GR, Medeiros H, Vyas JM, Wang Y, Hooper DC. Role of the Tet38 efflux pump in staphylococcus aureus internalization and survival in epithelial cells. Infect Immun. 2015;83(11):4362-72. PMID: 26324534 DOI: 10.1128/IAI.00723-15

9. Becker K, Denis O, Roisin S, Mellmann A, Idelevich EA, Knaack D, et al. Detection of mecA- and mecC-positive methicillin-resistant staphylococcus aureus (MRSA) isolates by the new xpert mrsa gen 3 PCR assay. J Clin Microbiol. 2016;54(1):180-4. PMID: 26491186 DOI: 10.1128/JCM.02081-15

10. Wang S, Wang Y, Shen J, Wu Y, Wu C. Polymorphic mutation frequencies in clinical isolates of Staphylococcus aureus: the role of weak mutators in the development of fluoroquinolone resistance. FEMS Microbiol Lett. 2013;341(1):13-7. PMID: 23330696 DOI: 10.1111/15746968.12085

11. Tahmasebi H, Bokaeian M, Adabi J. Coagulase-negative, beta-lactam, antibiotic resistance, methicillin resistance. Pars J Med Sci. 2016;14(1):55-63. [Persian]

12. Bokaeian M, Adabi J, Zeyni B, Tahmasebi H. The presence of aac (6 ') Ie / aph (2 "), aph (3') - IIIa1, ant (4 ') - Ia1 genes and determining methicillin resistance in staphylococcus epidermidis and staphylococcus saprophyticus strains isolated from clinical specimens. Arak Med Univ J. 2017; 19(11):11-25. [Persian]

13. CLSI. M100-S25 performance standards for antimicrobial

$$
\begin{aligned}
& \text { داراى مقاومت جند گانه تظاهر يِيدا مى كنند، مى توانند با استفاده }
\end{aligned}
$$

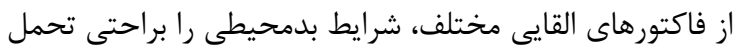

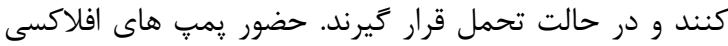

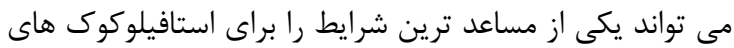

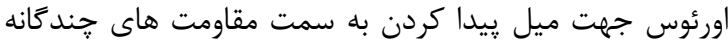

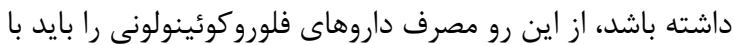

$$
\begin{aligned}
& \text { الخوى مناسب ترى مصرف كرد. }
\end{aligned}
$$

susceptibility testing. CLSI MI00-S20. Wayne, PA: Clinical and Laboratory Standards Institute; 2015.

14. Truong-Bolduc QC, Hooper DC. The transcriptional regulators NorG and MgrA modulate resistance to both quinolones and $\beta$-Lactams in staphylococcus aureus. $J$ Bacteriol. 2007;189(8):2996-3005. PMID: 17277059 DOI: 10.1128/JB.01819-06

15. Costa SS, Viveiros M, Amaral L, Couto I. Multidrug efflux pumps in Staphylococcus aureus: an update. Open Microbiol J. 2013;6(7):59-71. PMID: 23569469 DOI: $10.2174 /$ 1874285801307010059

16. Arabestani MR, Alikhani MY, Karami M, Salimi Ghale E. Prevalence of coagulase-negative staphylococci and determination of antimicrobial resistance in accompany with types of SCCmec in isolated of nosocomial infections. Tehran Univ Med J. 2016:73(12):888-94. [Persian]

17. Ahmadi Z, Tajbakhsh E, Momtaz H. Detection of the antibiotic resistance pattern in Staphylococcus aureus isolated from clinical samples obtained from patients hospitalised in Imam Reza hospital, Kermanshah. J Microb World. 2014;6(4):299-311. [Persian]

18. Vola ME, Moriyama AS, Lisboa R, Vola MM, Hirai FE, Bispo PJ, et al. Prevalence and antibiotic susceptibility of methicillin-resistant Staphylococcus aureus in ocular infections. Arq Bras Oftalmol. 2013;76(6):350-3. PMID: $\underline{24510081}$

19. Kang CY, Chaudhry OO, Halabi WJ, Nguyen V, Carmichael JC, Mills S, et al. Risk factors for postoperative urinary tract infection and urinary retention in patients undergoing surgery for colorectal cancer. Am Surg. 2012;78(10):1100-4. PMID: 23025950

20. Sierra JM1, Marco F, Ruiz J, Jiménez de Anta MT, Vila J. Correlation between the activity of different fluoroquinolones and the presence of mechanisms of quinolone resistance in epidemiologically related and unrelated strains of methicillinsusceptible and -resistant Staphylococcus aureus. Clin Microbiol Infect. 2002;8(12):781-90. PMID: 12519351

21. Kazemi SS, Nemati MF, Mirzaie A, Ashrafi F. Antibiotic resistance assessment, and genotypic and phenotypic detection of norA efflux pump in methicillin and ciprofloxacin resistant Staphylococcus aureus isolates. $J$ Microb World. 2017;9(4):286-96. [Persian]

22. Santos Costa S, Viveiros M, Rosato AE, Melo-Cristino J, Couto I. Impact of efflux in the development of multidrug resistance phenotypes in Staphylococcus aureus. BMC Microbiol. 2015;15(1):232-45. PMID: 26498754 DOI: 10.1186/s12866-015-0572-8

23. Blanco P, Hernando-Amado S, Reales-Calderon JA, Corona F, Lira F, Alcalde-Rico M, et al. Bacterial multidrug efflux pumps: much more than antibiotic resistance determinants. Microorganisms. 2016;4(1):14. PMID: 27681908 DOI: 10.3390/microorganisms4010014

24. Sun J, Deng Z, Yan A. Bacterial multidrug efflux pumps: mechanisms, physiology and pharmacological exploitations. Biochem Biophys Res Commun. 2014;453(2):254-67. PMID: 24878531 DOI: $10.1016 /$ j.bbrc. 2014.05 .090

25. Martineau F, Picard FJ, Lansac N, Ménard C, Roy PH, Ouellette $\mathrm{M}$, et al. Correlation between the resistance genotype determined by multiplex PCR assays and the antibiotic susceptibility patterns of staphylococcus aureus and staphylococcus epidermidis. Antimicrob Agents Chemother. 2000;44(2):231-38. PMID: 10639342

26. Costa SS, Falcão C, Viveiros M, Machado D, Martins M, 
Melo-Cristino J, et al. Exploring the contribution of efflux on the resistance to fluoroquinolones in clinical isolates of Staphylococcus aureus. BMC Microbiol. 2011;11(9):110-29. PMID: 22032541 DOI: 10.1186/1471-2180-11-241

27. Ranjbarian P, Sadeghian S, Shirazi MH, Sarafnezhad A,
Fazeli MR, Amin GH, et al. Survey of anti-bacterial effect of plant extracts (fennel-dill-caraway-cinnamon) by flow cytometry and disk diffusion. Sci J Hamadan Univ Med Sci Health Ser. 2004;11(3):42-7. [Persian] 